\title{
Neonatal Outcomes from Mothers with Hypertension Disorders of Pregnancy: A Retrospective Study at a Referral Hospital in Rwanda
}

\author{
Phoibe Uwizeyimana1,2*, Emerthe Musabyemariya ${ }^{2}$, Olive Tengera $^{2}$, Anita Collins ${ }^{3}$ \\ ${ }^{1}$ Butare University Teaching Hospital, Huye, Rwanda \\ ${ }^{2}$ School of Nursing and Midwifery, University of Rwanda, College of Medicine and Health Sciences, \\ Kigali, Rwanda. \\ ${ }^{3}$ Rory Meyer's College of Nursing, New York University, New York, USA \\ *Corresponding author: Phoibe Uwizeyimana. Butare University Teaching Hospital, P.O. Box \\ 254 Huye, Rwanda, Email: uphoibe@gmail.com
}

\begin{abstract}
Background

Globally, maternal hypertensive disorders in pregnancy significantly increase both maternal and perinatal morbidity and mortality. Maternal hypertension affects 14 percent of pregnancies. Eearly detection and management are critical for improving the health outcomes of both mother and neonate.
\end{abstract}

\section{Objective}

To assess the association between maternal hypertension disorders in pregnancy and immediate neonatal outcomes at a University Teaching Hospital in Rwanda.

\section{Methods}

A retrospective study of maternal files with hypertension disorders was conducted from January 1, 2016, to March 31, 2019. A census sample of 114 records and pretested checklist was used to collect data. Descriptive statistics were used to analyze associations between maternal factors and immediate neonatal outcomes.

\section{Results}

Neonatal outcomes included low birth weight (75.4\%), prematurity (59.6\%), admission to neonatal intensive care unit (50.4\%), intrauterine growth restriction (32.4\%), and neonatal death $(22.8 \%)$. Nearly two-thirds $(62 \%)$ of mothers had preeclampsia. Significant associations with immediate neonatal outcomes included gestational age, medical history, delivery mode, maternal referral status, preterm birth, prematurity, and abortion.

\section{Conclusion}

Maternal hypertensive disorders were significantly associated with adverse neonatal outcomes in our study population in Rwanda. Improving early detection, health education, and management of hypertensive disorders in pregnancy is critical to reduce maternal and neonatal morbidity and mortality.

Rwanda J Med Health Sci 2020;3(2):193-203

Keywords: Neonatal outcomes, maternal hypertensive disorders, hypertension 


\section{BACKGROUND}

Globally, maternal and perinatal morbidity and mortality due to maternal hypertension in pregnancy is a significant public health threat in low-and middle-income countries (LMIC) and highincome countries.[1] A systematic review of 115 countries worldwide revealed that $14 \%$ of all maternal mortality was due to hypertensive pregnancy disorders, amounting to 42,000 deaths annually.[1,2] Furthermore, almost 1 in 5 (17.9\%) hypertensive women had a stillbirth or neonatal death in a study in sub-Sahara Africa (SSA), India, and Haiti.[3]

Maternal hypertensive disorders are more likely to cause significant adverse perinatal outcomes in LMIC. It is estimated that 2.6 million stillbirths occur each year, and 98\% occur in LMIC.[4] Other serious neonatal outcomes from maternal hypertension include prematurity, low birth weight (LBW), intrauterine fetal demise (IUFD), intrauterine growth restriction (IUGR), respiratory distress, admission at neonatal intensive care unit (NICU), and neonatal death.[5]

Hypertension in pregnancy is defined as either a systolic blood pressure (BP) of $\geq 140 \mathrm{~mm} \mathrm{Hg}$ OR a diastolic $\geq 90 \mathrm{~mm} \mathrm{Hg}$.[6] Maternal hypertensive disorders are classified into four types: Chronic hypertension, that is known before pregnancy, or present in the first 20 weeks. New onset of hypertension that develops at or after 20 weeks gestation includes preeclampsia superimposed on chronic hypertension, preeclampsia, and eclampsia and gestational hypertension.[6] Among these four hypertensive disorders, preeclampsia and eclampsia have the most significant effects on maternal and newborn morbidity and mortality.[6] However, timely and effective interventions using evidence-based practice could prevent morbidity and mortality.[7]

Numerous studies are addressing hypertensive disorders in pregnancy. A systematic review and meta-analysis of 55 studies in the United States [6] reported pregnant women $(\mathrm{n}=795221)$ with chronic hypertension had a higher incidence of superimposed preeclampsia (25.9\%), a cesarean delivery $(41.4 \%)$, preterm birth at 37 weeks or less $(28.1 \%)$ of $<2500$ grams $(16.9 \%)$ NICU care $(20.5 \%)$ and perinatal death (4.0\%).[8]

In SSA, perinatal loss rates are higher than in highincome countries. In South Africa, a prospective study of pregnant women (n=1547) with preeclampsia at three tertiary facilities, had 1589 births (including 42 twins), revealed a $21 \%$ perinatal mortality rate, and of this $84.5 \%$ were stillbirths.[9] Of the live births $(n=1308), 70.0 \%$ were born preterm $(<37$ weeks), and of those, $41.7 \%$ were born at $<34$ weeks' gestation.[9] In Southern Ethiopia, a retrospective study of women $(n=7347)$ hospitalized with a hypertensive disorder reported a $49 \%$ preterm birth rate.[9] In Uganda, a cross-sectional study compared adverse neonatal outcomes with good neonatal outcomes, and found pregnant women with preeclampsia were nearly six times more likely to have a preterm birth (OR 5.97).[10]

The Rwanda Demographic Health Survey (RDHS), 2014-2015 showed that neonatal and post-neonatal mortality rates were at 20 deaths per 1,000 live births and 13 deaths per 1,000 live births, respectively.[11] The perinatal mortality rate was 29 deaths per 1,000 pregnancies.[11]

Since maternal hypertensive disorders are a significant public threat to both mother and newborn, a study was undertaken to identify the existing situation of neonatal outcomes in Rwanda. Neonatal outcomes are commonly cited in the perinatal literature; however, the outcome has not been reported concerning the mother's hypertensive disorder in many cases. A specific look at the University Teaching Hospital of Butare (CHUB) revealed a high incidence of hypertensive disorders, with about 7.6 cases per month among the 350 patients admitted.[12] The scarcity of evidence about this area of study in Rwanda inspired the investigator to assess the neonatal outcome of mothers with hypertensive disorders to improve the management of neonates by reducing fetal and neonatal mortality through timely delivery and appropriate care. This study aimed to identify immediate outcomes of neonates from mothers with hypertension disorders and associated maternal factors.

\section{METHODS}

\section{Design}

A descriptive retrospective study design of mothers' records and files was used to determine neonates' immediate outcomes from mothers who had hypertension during pregnancy. The study site was at CHUB, in Rwanda. 


\section{Participants' recruitment}

A purposive strategy and a census sampling method (also known as complete enumeration) were used. Data were collected from all available patients' files delivered by mothers with hypertensive disorders during the study period from January 1, 2016, to March 31, 2019. A total of 114 neonates were eligible and selected for the study. All neonates delivered by mothers with hypertensive disorders and included: chronic HTN, preeclampsia superimposed on chronic HTN, preeclampsia and eclampsia, and gestational HTN (hypertension).

\section{Measures}

The checklist was developed from the published literature and included the conceptual framework for adverse birth outcomes developed by Olusanya and Ofovwe in Nigeria.[11]

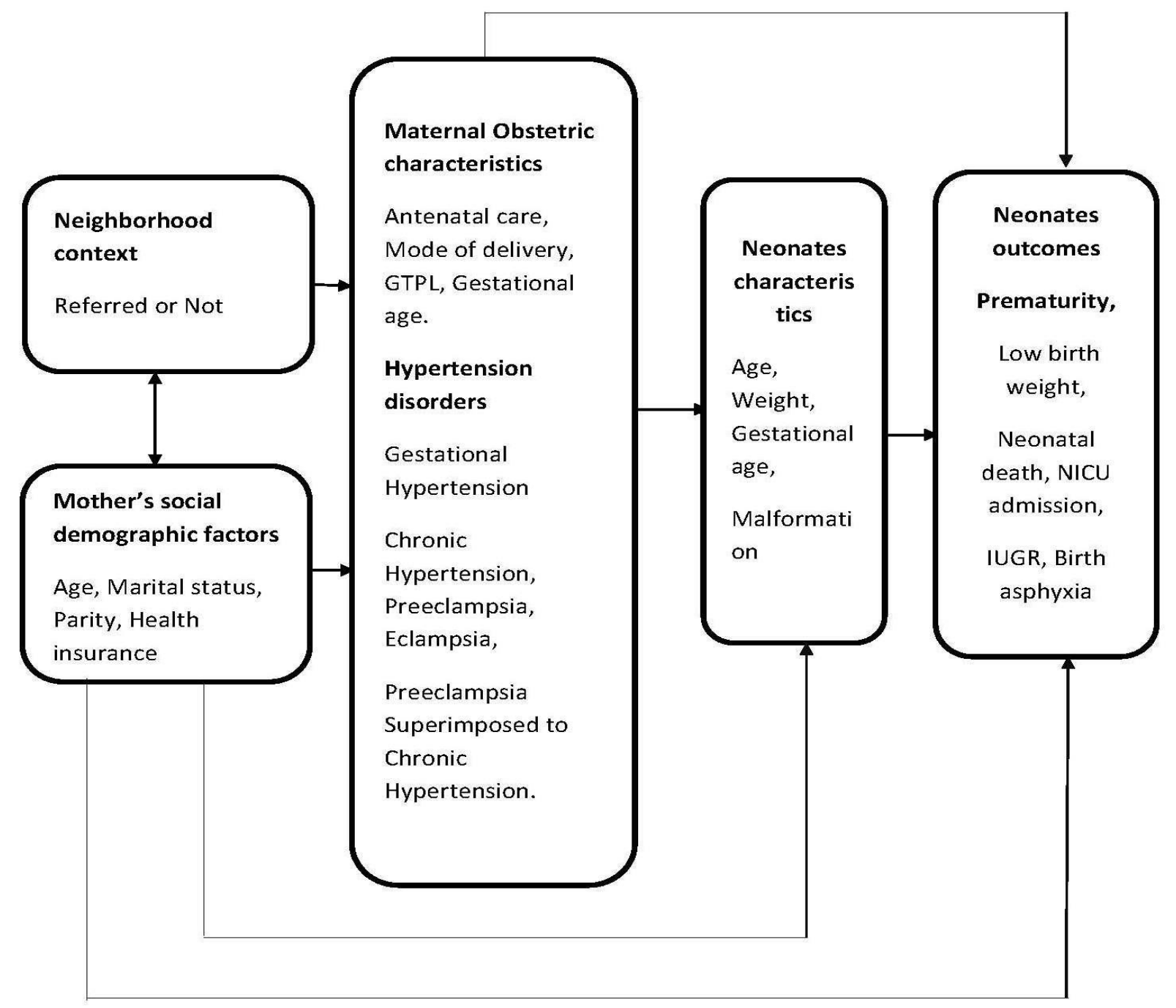

Figure 1: Study conceptual Framework for neonatal Birth Outcomes adapted Olusanya

and Ofovwe, 2010 p.409-415)*. [12]

*Adapted from Olusanya and Ofovwe, (2010, p.409-415) [13] 
The checklist consisted of three parts:

The first part comprised of sociodemographic characteristics (4 items). Gestational age (abortion $[<20$ weeks], preterm $[<37.6$ weeks], term $[\geq 38$ weeks]). Medical history: HTN (chronic HTN; preeclampsia superimposed on chronic HTN; preeclampsia and eclampsia; gestational HTN); IUFD; and other conditions. Mode of delivery (spontaneous vaginal delivery [SVD] or Cesarean delivery) maternal referral. Variables were presented in frequencies

The second part comprised of the neonatal parameters and status (10 items), and included congenital malformation; IUGR; neonatal weight (< 2500 grams, 2500-4000 g); NICU admission; meconium aspiration syndrome; transient tachypnea of the newborn (TTN); respiratory distress (mild, moderate and severe); neonatal demise (death, no death); APGAR at 1, 5, and 10 minutes. The Apgar classification of asphyxia at 10 min was mild (5-6), moderate (4-3), and severe (2$0)$. Variables were scored as frequencies.

The third part was composed by neonatal outcomes (five items) and included gestational age, neonate birth weight, asphyxia, NICU admission, and neonatal death. Chi-square was used to identify associations

The data collection checklist was pretested on ten maternal patients' files to test for the feasibility of the study, and these files were not included in the final data. The results of this pretest suggested, minor correction, of the checklist before final data collection. Items that were removed included the item on antenatal booking, maternal occupation, and educational attainment, as these items were not documented in patients' files.

\section{Data collection}

The data were collected by the investigator and two well-trained research assistants (RA). The investigator and RAs visited the hospital archives and selected the patients' numbers corresponding to the mothers' records with hypertensive disorders. All retrieved files were kept alone for the exploration of neonates and maternal parameters.

\section{Data analysis}

The data collected were coded and entered into SPSS, version 21. Descriptive statistics such as mean, standard deviation, and frequencies tables were used to summarize the data. Chi-square test was used to assess the association between maternal factors sociodemographic variables with the neonatal outcome parameters of prematurity, asphyxia, NICU admission, and neonatal death. The significance was set at $\mathrm{p}<0.05$.

\section{Ethical considerations}

The Institutional Review Board, of the College of Medicine and Health Sciences, at the University of Rwanda, approved the study. Also, the CHUB Research and Ethics Committee granted permission to use archives and collect the data. We used individual patient numbers/codes to ensure the anonymity of the study participants.

\section{RESULTS}

This retrospective study assessed the immediate neonatal outcomes among 114 mothers with hypertensive disorders in pregnancy during a thirtynine-month data collection period. The maternal sociodemographic characteristics are presented in Table 1 . 


\begin{tabular}{lc}
\hline Table 1. Maternal sociodemographic charact \\
\hline Characteristics & $\mathbf{n}(\%)$ \\
\hline Mode of delivery & \\
SVD & $59(51.8)$ \\
Cesearean & $55(48.2)$ \\
Past Medical History & \\
HTN & $39(34.2)$ \\
IUFD & $19(16.7)$ \\
Other & $56(49.1)$ \\
Maternal Referral^(n=102) & \\
Yes & $31(30.4)$ \\
No & $71(69.6)$
\end{tabular}

$\wedge$ Missing data

The sociodemographic characteristics of the mothers indicated that over half $(51.8 \%)$ had a SVD, and nearly half $(48.2 \%)$ had a cesarean delivery (Table 1$)$. Over one-third (34.2\%) had a past medical history of hypertension. Nearly a third (30.4\%) of mothers were referred from the district's hospitals to the CHUB.

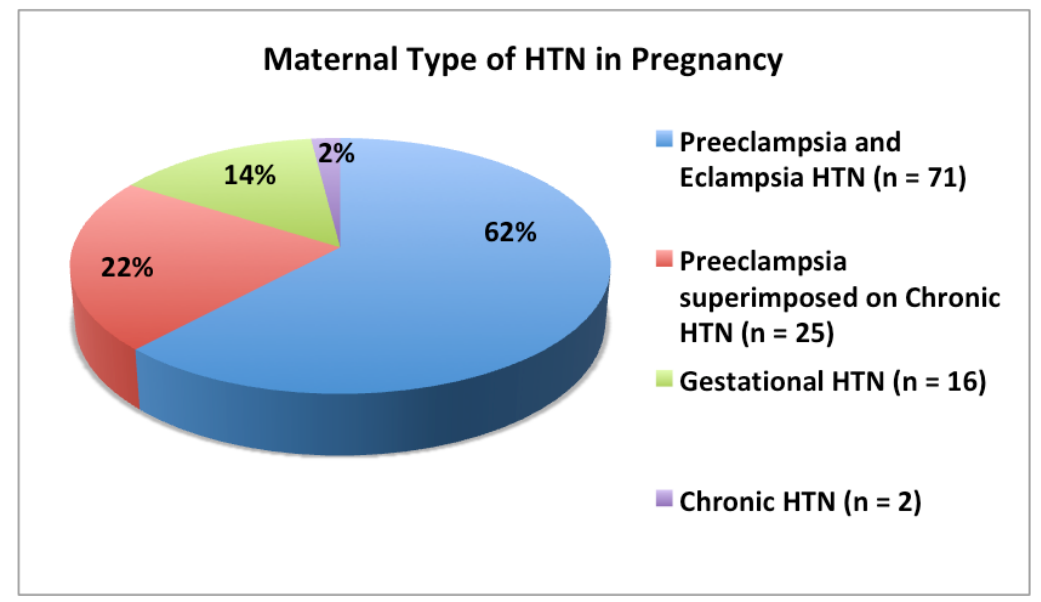

Figure 2. Maternal type of HTN disorder

Nearly two thirds (62\%) of the mothers had preeclampsia and eclampsia HTN during pregnancy (Figure 1). Other types included preeclampsia superimposed on chronic HTN $(22 \%)$, gestational HTN (14\%), and chronic HTN $(2 \%)$. 
Table 2. Immediate parameters and status of neonates

\begin{tabular}{|c|c|}
\hline Variables & n (\%) \\
\hline \multicolumn{2}{|l|}{ Newborn } \\
\hline $2500-4000 \mathrm{~g}$ & $28(24.6)$ \\
\hline \multicolumn{2}{|l|}{ Gestational age } \\
\hline Abortion & $12(10.5)$ \\
\hline Preterm & $68(59.6)$ \\
\hline Term & $34(29.8)$ \\
\hline \multicolumn{2}{|l|}{ IUGR } \\
\hline Yes & $36(32.4)$ \\
\hline \multicolumn{2}{|c|}{ Congenital malformation } \\
\hline Yes & 7 (6.4) \\
\hline \multicolumn{2}{|c|}{ Meconium aspiration syndrome } \\
\hline Yes & $19(17.4)$ \\
\hline \multicolumn{2}{|l|}{ Respiratory distress } \\
\hline Mild & $15(13.2)$ \\
\hline Moderate & $14(12.3)$ \\
\hline Severe & $14(12.3)$ \\
\hline TTN & 17 (14.9) \\
\hline None & $54(47.4)$ \\
\hline \multicolumn{2}{|l|}{ Neonatal weight } \\
\hline$<2500 \mathrm{~g}(\mathrm{LBW})$ & $86(75.6)$ \\
\hline 2500-4000 g (Normal) & $28(24.6)$ \\
\hline \multicolumn{2}{|l|}{ NICU admission } \\
\hline Yes & $57(50.4)$ \\
\hline No & $56(49.6)$ \\
\hline \multicolumn{2}{|l|}{ Neonatal death } \\
\hline Yes & $26(22.8)$ \\
\hline No & $88(77.2)$ \\
\hline
\end{tabular}

The majority of neonates were premature (59.6\%), and term births were a lower proportion (29.8\%) (Table 2). Nearly a third (32.4\%) of neonates had IUGR. The APGAR score shows severe asphyxia at 1 min (38.6\%), $5 \mathrm{~min}(30.7 \%)$, and $10 \mathrm{~min}(28.9 \%)$. Neonates with no asphyxia had the following proportions at $1 \mathrm{~min}$ (34.2\%), $5 \mathrm{~min}(43.9 \%)$, and $10 \mathrm{~min}(50.0 \%)$. Over half $(50.4 \%)$ of neonates were admitted to the NICU, and nearly a quarter died $(22.8 \%)$.

Table 3. APGAR score at three time points $(n=114)$

\begin{tabular}{|c|c|c|c|}
\hline APGAR & $\begin{array}{l}1 \text { min } \\
\mathrm{n}(\%)\end{array}$ & $\begin{array}{l}5 \text { min } \\
n(\%)\end{array}$ & $\begin{array}{l}10 \text { min } \\
\mathrm{n}(\%)\end{array}$ \\
\hline \multicolumn{4}{|l|}{ Asphyxia } \\
\hline Severe & $44(38.6)$ & 35 (30.7) & 33 (28.9) \\
\hline Moderate & $20(17.5)$ & 15 (13.1) & $10(8.8)$ \\
\hline Mild & $11(9.7)$ & 14 (12.3) & 14 (12.3) \\
\hline None & $39(34.2)$ & 50 (43.9) & $57(50.0)$ \\
\hline
\end{tabular}

The APGAR scores taken immediately after birth are presented in Table 3. The APGAR at 1 min shows that nearly a third of neonates had severe asphyxia (30.7\%), at $5 \mathrm{~min}(30.7 \%)$, and $10 \mathrm{~min}(28.9 \%)$. Neonates with no asphyxia were $34.2 \%, 43.9 \%$, and $50.0 \%$ at $1 \mathrm{st}, 5^{\text {th }}, 10^{\text {th }}$ min post-birth, respectively. 
Table 4. Significant associations between maternal factors and neonatal outcomes

\begin{tabular}{llll}
\hline Variables & Yes & No & p-value \\
& $\mathbf{n}(\%)$ & $\mathbf{n}(\%)$ &
\end{tabular}

\section{Maternal factor \\ Referral (n=31) \\ Gestational Age \\ Preterm \\ Term}

\section{Neonatal Factors \\ Low Birth Weight \\ Gestational age}

Abortion

Preterm

Term

Asphyxia

Past medical history

HTN

IUFD

Other

Mode of delivery

SVD

Cesarean

NICU Admission

Past medical history

HTN

IUFD

Other

Mode of delivery

SVD

Cesarean

Gestational age

Abortion

Preterm

Term

Past Medical History

HTN
IUFD
Other
Sode of delivery
SVD
Cesarean

\section{Gestational age}

Abortion

Preterm

Term
Neonatal Death

$\begin{array}{lll}14(45.2) & 17(54.8) & \\ 17(54.8) & 14(54.8) & 0.002^{* *}\end{array}$

$\begin{array}{lll}12(100.0) & 0(0) & \\ 60(88.2) & 8(11.8) & 0.001^{*} \\ 14(41.2) & 20(58.8) & \end{array}$

$16(41.0) \quad 23(59.0)$

$3(15.8) \quad 16(84.2) \quad 0.006^{* *}$

$32(57.1) \quad 24(42.9)$

$19(32.2) \quad 40(67.8)$

32(58.2) $23(41.8) \quad 0.005^{* *}$

$\begin{array}{lll}16(41.0) & 23(59.0) & \\ 3(15.8) & 16(84.2) & 0.000^{* * *} \\ 39(69.6) & 17(30.4) & \\ & & \\ 22(37.3) & 37(62.7) & \\ 36(65.5) & 19(34.5) & 0.003^{* *} \\ 4(33.3) & 8(66.7) & \\ 43(63.2) & 25(36.8) & 0.006^{* *} \\ 11(32.4) & 23(67.6) & \end{array}$

$\begin{array}{lll}8(20.5) & 31(79.5) & \\ 15(78.9) & 4(21.1) & 0.000 * * * \\ 3(5.4) & 53(94.6) & \\ & & \\ 23(39.0) & 36(61.0) & \\ 3(5.5) & 52(94.5) & 0.000^{* * *} \\ & & \\ 9(75.0) & 3(25.0) & \\ 14(20.6) & 54(79.4) & 0.000^{* * *} \\ 3(8.8) & 31(91.2) & \end{array}$

The significant associations between the maternal factors and neonatal outcomes are presented in Table 4 


\section{Maternal referral}

The findings show that referral was the only maternal factor that was significantly associated with gestational age at birth $(\mathrm{p}=0.002)$. Approximately half of the term $(54.8 \%)$ and preterm newborns $(45.2 \%)$ had a mother with a hypertensive disorder, and mothers were referred from district hospitals to the University Teaching Hospital of Butare.

\section{Neonatal birth weight}

The findings show that LBW was significantly associated with gestational age at birth $(\mathrm{p}=0.001)$ among a mother who had a hypertensive disorder. The majority $(88.2 \%)$ of preterm newborns and nearly half $(41.2 \%)$ of term newborns had low-birth weight.

\section{Asphyxia}

Two variables were significantly associated with asphyxia, including maternal medical history $(\mathrm{p}=$ $0.006)$ and mode of delivery $(p=0.005)$. Newborns whose mothers had a past medical history of HTN $(41 \%)$, an IUFD $(15.8 \%)$, and other conditions $(57.1 \%)$ had asphyxia. Furthermore, nearly a third $(32.2 \%)$ of SVD births and over half $(58.2 \%)$ of cesarean deliveries were diagnosed with asphyxia around the time of birth.

\section{NICU Admission}

Three variables were significantly associated with NICU admissions, and include maternal medical history $(p=0.001)$, delivery mode $(p=0.003)$, and gestational age $(p=0.006)$. Newborns whose mothers had a past medical history of HTN $(41.0 \%)$, IUFD $(15.8 \%)$, and other conditions $(69.6 \%)$ were associated with NICU admissions. Also, two thirds $(65.5 \%)$ of cesarean deliveries and preterm births $(63.2 \%)$ were significantly associated with NICU admissions.

\section{Neonatal death}

Three variables were significantly associated with neonatal death, and include maternal medical history, $(p=0.000)$ mode of delivery $(p=0.000)$, gestational age $(p=0.001)$. Newborns whose mothers had a past medical history of HTN $(20.5 \%)$, IUFD $(78.9 \%)$, and other conditions $(5.4 \%)$ were associated with neonatal death. Also, over one third $(39.0 \%)$ of SVD births and nearly a quarter $(20.6 \%)$ of preterm newborns were significantly associated with neonatal death.

\section{DISCUSSION}

This retrospective study assessed the association between the immediate outcomes of 114 neonates whose mothers had experienced a hypertension disorder during pregnancy. Five neonatal outcomes; gestational age, neonate birth weight, asphyxia, NICU admission, and neonate death were assessed for associations with maternal hypertension.

\section{Immediate parameters and status of neonates}

The analysis of neonates' parameters showed that the APGAR scores revealed that $50 \%$ of neonates had asphyxia, and $50.4 \%$ were admitted to NICU. In addition, $75.4 \%$ had LBW and $22.8 \%$ died. Similar findings have been reported in many studies where was common and many neonates died that were born to mothers with hypertension. The same study further reported an APGAR score below 7 among $38.6 \%$ of neonate at $5 \mathrm{~min}$, indicating asphyxia.[14$16]$

The study done in Ethiopia [17] showed that still birth, LBW, abortion, preterm birth and low APGAR score were the most neonatal outcomes in mothers with hypertension most common neonatal outcomes disorders. Abortion and preterm delivery were into the proportions of $10.7 \%$ and $31.4 \%$ respectively. Similar studies from both LMIC and high-income countries also revealed that children from mothers with hypertension disorders had an increased risk for admission to the NICU as compared with children from normotensive mothers. Also, our findings concur with those from another study in Saudi Arabia [18] on the prevalence, risk factors, and fetal and maternal outcomes of mothers with hypertensive disorders of pregnancy .

The study concluded that there was a higher number of preterm, IUGR and SGA babies among the infants from hypertensive mothers. The same study further suggested that pregnancy induced hypertension (PIH) or hypertensive disorders are one of the most common causes of both maternal and neonatal morbidity.[18]

\section{Outcomes of neonates and associated maternal factors.}

In this study, past medical history of hypertension shows a strong association with the NICU admission (P-value: $0.001<0.05)$, neonate asphyxia (P-value: $0.006<0.05)$, neonate death (P-value: $0.001<0.05$ ). Other factors that show a linkage with the 
admission include gestational age and mode of delivery (P-values: 0.006, and 0.003 respectively), they both show a significant association with neonatal death $(\mathrm{P}$-value: $0.001<0.05)$.

Many studies reported that hypertension during pregnancy increases the risk of maternal and perinatal, morbidity and mortality.[5-7] Comparable findings were observed in a study from Queen Elizabeth Central Hospital (QECH) neonatal ward records where $36.5 \%$ of neonates admitted in the ward from April to September 2012 had birth asphyxia.[21]

A study by Ahmed and colleagues revealed a frequency of perinatal asphyxia varied from $9 \%$ to $22 \%$ in different studies.[22] In this study, delivery mode was also linked with birth asphyxia (P-value: $0.005<0.05)$. Likewise, Kibitui found out that linkage and the association between those two variables was strong.[23] It is also important to remember that in the management of hypertensive disorder, an emergency caesarean section must be done to save maternal and foetal life. This has also implication for NICU admission since the baby may have, LBW, IUGR, prematurity, low APGAR score $(<7)$ and birth asphyxia as was observed in half of our study sample group. Asphyxia also predisposes neonates to death. Our results about gestational age are in support with the findings from a Tertiary Care Centre from India which concluded that 54.67\% neonates born to hypertensive mothers were low birth weight and over 63\% newborn were preterm.[3] Another study mentioned that the cause of neonatal mortality is due to prematurity and LBW, and that NICU admissions and duration of stay was high.[24]

Some hospital based prospective and retrospective studies from Nepal and Ethiopia showed that respective proportions of $13 \%$ and $30.2 \%$ of babies were delivered premature.[25] These are in line with our findings, though reported proportions of prematurity were comparatively low. This discrepancy resides in the methods and enrolled pregnant women into research studies.

In our study, hypertension disorders were mainly preeclampsia, eclampsia and chronic hypertension. This has also been the reason for the high proportion in neonatal outcomes as compared to other studies.[25,26]

\section{Limitations}

There are two main limitations to this study that could be addressed in future research. First, the method used in this study was retrospective; therefore, we were limited to the data previously collected, and some of the data on the quality of care given to neonates was missing from the files. Secondly, there was a small sample size due to the time constraint of data collection.

\section{Recommendations}

We recommend early recognition and best time to deliver management and treatment of hypertensive disorders. We recommend reducing neonatal deaths due to birth asphyxia through quality service improvement in maternity and neonatology departments. Significant efforts should be directed to close monitoring and management of mothers with hypertensive disorders to save the lives of mothers, fetuses, and neonates. Moreover, maternal and child HCPs at all levels need to identify women at risk, by optimizing management of pre-existing maternal medical conditions such as hypertension, diabetes, and obesity; and offer counseling, closer monitoring, and refer as needed to a hospital for antihypertensive medications that are considered safe in pregnancy. WHO recommends calcium supplementation before pregnancy for the prevention of preeclampsia and its complications.[27] This combined effort may, in turn, reduce the maternal and neonatal morbidity and mortality associated with preeclampsia and other HTN disorders in pregnancy.

\section{CONCLUSION}

This retrospective study of a population at a referral hospital in Rwanda demonstrated that there is a relationship between maternal hypertensive disorders and neonatal outcomes. Neonatal adverse events such as asphyxia, LBW, prematurity, IUGR, NICU admission, and neonatal death were more prevalent if the mother had a hypertensive disorder during pregnancy. Additional studies are needed to understand the causal pathways that may cause disordered fetal development in preeclampsia, as well as the adult disease processes a future study is needed in predisposing factors of developing gestational hypertension in women who use $\geq 800$ $\mu \mathrm{g} / \mathrm{d}$ FA as a supplement during pregnancy.

\section{Author's contribution}

PU was the principal investigator for whole process from data collection to final manuscript. ME reviewed the checklist used in data collection, OT was the co-supervisor who reviewed and revised the article. AC was the supervisor. AC was the supervisor by coordinating all process in writing, developing the manuscript. All authors read and approved the final manuscript.

\section{Conflict of interest declaration}

The authors report that here was no conflict of 
interest to this article

\section{Acknowledgments}

Authors would like to thank Dr. Pamela Meharry this publication through the writing retreats among neonatal students and manuscript development.

This article is published open access under the Creative Commons Attribution-NonCommercial NoDerivatives (CC BYNCND4.0). People can copy and redistribute the article only for noncommercial purposes and as long as they give appropriate credit to the authors. They cannot distribute any modified material obtained by remixing, transforming or building upon this article. See https://creativecommons.org/licenses/by-ncnd/4.0/

\section{REFERENCES}

1. Vousden N, Lawley E, Seed PT, Gidiri MF, Goudar S, Sandall J, et al. Incidence of eclampsia and related complications across 10 low-and middlere source geographical regions: Secondary analysis of a cluster randomised controlled trial. PLoS Med. 2019;16:1-15.

2. Berhan Y, Endeshaw G. ORIGINAL ARTICLE MATERNAL MORTALITY PREDICTORS IN WOMEN WITH HYPERTENSIVE DISORDERS OF PREGNANCY : RETROSPECTIVE COHORT STUDY.

3. Saini S, Singh M, Mittal A. A study to assess the association of maternal factors and the outcome of pregnancy among mothers delivering in a tertiary care hospital in Haryana, India. Int $\mathrm{J}$ Community Med Public Heal. 2016;3:1716-22.

4. Subki AH, Algethami MR, Baabdullah WM, Alnefaie MN, Alzanbagi MA, Alsolami RM, et al. Prevalence, risk factors, and fetal and maternal outcomes of hypertensive disorders of pregnancy: A retrospective study in Western Saudi Arabia. Oman Med J [Internet]. 2018;33:409-15. Available from: https://www.google.com/search?q=Prevalence $\% 2 \mathrm{C}+$ risk + factors $\% 2 \mathrm{C}+$ and + fetal+and+maternal+outcomes+of+hypertensive+disorders+of+pregnancy $\% 3 \mathrm{~A}+\mathrm{A}+$ retrospective+study+in+Western+Saudi+Arabia\&rlz=1C1GGRV_enRW783RW783\&oq=Pr evalence $\% 2 \mathrm{C}+$ risk+factors $\% 2 \mathrm{C}+$ and + fetal+and $+m$

5. Endeshaw G, Berhan Y. Perinatal Outcome in Women with Hypertensive Disorders of
Pregnancy: A Retrospective Cohort Study. 2015;2015.

6. Hassan M, Begum M, Haque SMZ, Jahan N, Yasmeen BHN, Mannan A, et al. Immediate Outcome of Neonates with Maternal Hypertensive Disorder of Pregnancy at a Neonatal Intensive Care Unit. 2015;6:57-60.

7. Obsa MS, Woticha EW, Weji BG, Dessu BK, Wolde GD, Girmay BG, et al. Neonatal and Fetal Outcomes of Pregnant Mothers with Hypertensive Disorder of Pregnancy at Hospitals in Wolaita. J midwefery Reprod Heal [Internet]. 2019;2:1-6. Available from: http://jmrh.mums.ac.ir/arti-

cle_12073_aedf781274516c2aa855fd8ce9b 3b940.pdf

8. Brown MA, Magee LA, Kenny LC, Karumanchi SA, McCarthy FP, Saito S, et al. Hypertensive disorders of pregnancy: ISSHP classification, diagnosis, and management recommendations for international practice. Hypertension. 2018;72:24-43.

9. Nathan HL, Paul T, Greeff A De, Conti- F, Anthony J, Steyn W, et al. Maternal and perinatal adverse outcomes in women with preeclampsia cared for at facility-level in South Africa : a prospective cohort study. 2018;8.

10. Kiondo P, Tumwesigye NM, Wandabwa J, Wamuyu-maina G, Bimenya GS, Okong P. Supplement article Adverse neonatal outcomes in women with pre- eclampsia in $\mathrm{Mu}-$ lago Hospital, Kampala, Uganda: a crosssectional study. 2014;17:1-5.

11. MINISANTE. Rwanda Demographic and Health Survey 2014-2015. 2014.

12. MOH. Health Sector Annual Report July 2016-June 2017 0. 2017;11.

13. Olusanya BO, Ofovwe GE. Predictors of preterm births and low birthweight in an innercity hospital in sub-Saharan Africa. Matern Child Health J. 2010;14:978-86.

14. Melese MF, Badi MB, Aynalem GL. Perinatal outcomes of severe preeclampsia / eclampsia and associated factors among mothers admitted in Amhara Region referral hospitals, North West Ethiopia , 2018. BMC Res Notes [Internet]. BioMed Central; 2019;1-6. Available from: 
https://doi.org/10.1186/s13104-0194161-z

15. Perry JS. The Association Between Maternal Hypertensive Disorders and Perinatal Mortality in Kigoma Region, Tanzania: 20112015. 2018;2011-5.

16. Shrestha Pradhan S, Paudel S, Parajuli P, Rana Khagi B. Neonatal Outcome of Antenatal Mothers with Pregnancy Induced Hypertension in Antenatal Ward of Kathmandu Medical College and Teaching Hospital. Birat J Heal Sci. 2018;2:255-9.

17. Ibrahim NA, Muhye A, Abdulie S. Prevalence of Birth Asphyxia and Associated Factors among Neonates Delivered in Dilchora Referral Hospital, in Dire Dawa, Eastern Ethiopia. Clin Mother Child Heal. 2017;14.

18. Subki AH, Algethami MR. Prevalence, Risk Factors, and Fetal and Maternal Outcomes of Hypertensive Disorders of Pregnancy: A Retrospective Study in Western Saudi Arabia. 2018;33:409-15.

19. Siromani SM, Varahala AM, Gopu S, Chidugulla SK. Neonatal Outcome In Pregnancy Induced Hypertensive Mothers - A Tertiary Care Centre Experience. 2015;14:23-7.

20. Seyom E, Abera M, Tesfaye M, Fentahun N. Maternal and fetal outcome of pregnancy related hypertension in Mettu Karl Referral Hospital ,. J Ovarian Res [Internet]. 2015;1:4-10. Available from: https://www.researchgate.net/publication/274319447_Maternal_and_fetal_outcome_of_pregnancy_related_hypertension_in_Mettu_Karl_Referral_Hospital_Ethiopia
21. Tewesa E, Chirwa E, Majamanda MD, Maluwa A, Chimwaza A. Associative Factors for Birth Asphyxia at Queen Elizabeth Central Hospital - Malawi. 2017;22-31.

22. Ahmed SS, Sultana N, Begum ML, Lima LS, Abedin F. Pregnancy Induced Hypertension and Associated Factors among Pregnant Women. 2017;3.

23. Eric Kibitui K. Perinatal Factors Associated With Birth Asphyxia Among Neonates in Maternity Ward Kakamega County Refferal Hospital , Kenya. 2017;

24. Ejike DE, Ambrose B, Moses DA, Karimah MR, Iliya E, Sheu OS, et al. Determination, knowledge and prevalence of pregnancy-induced hypertension/eclampsia among women of childbearing age at Same District Hospital in Tanzania. Int J Med Med Sci. 2018;10:19-26.

25. Tesfaye A, Gudeta TA, Regassa TM. Pregnancy Induced Hypertension and Associated Factors among Women Attending Delivery Service at Mizan-Tepi University Teaching Hospital , Tepi General Hospital and Gebretsadik Shawo Hospital, Southwest, Ethiopia.

26. Doddamani UG, Doddamani GB. Perinatal Outcome in Pre-Eclampsia: A Prospective Study. 2014;2:291-3.

27. World Health Organization. WHO recommendation: Calcium supplementation during pregnancy for the prevention of pre-eclampsia and its complications [Internet]. 2018. Available from: http://www.ncbi.nlm.nih.gov/pubmed/30629391 